\title{
Review
}

\section{Helper-like Innate Lymphoid Cells in Humans and Mice}

\author{
Sophie Guia (i), ${ }^{1}$ and Emilie Narni-Mancinelli $\mathbb{1}{ }^{1,, *}$
}

The innate lymphoid cell (ILC) family consists of natural killer (NK) cells, helperlike lymphoid cells (ILC1s, ILC2s, and ILC3s), and lymphoid tissue inducer (LTi) cells. Helper-like ILCs are considered the innate counterpart of T-helper cells because of similarities in their cytokine output and expression of key transcription factors. ILCs provide and regulate innate immune functions before the development of adaptive immunity. They are involved in host defense against pathogens, inflammation, tissue repair, and metabolic homeostasis. However, they can also be involved in inflammatory disorders and carcinogenesis. In this review, we summarize the latest research on ILC development and plasticity in humans and mice, focusing on the pathogenic role of helper-like ILCs in inflammatory disorders, such as asthma, Crohn's disease (CD), and rheumatoid arthritis (RA).

\section{Innate Lymphoid Cells in Immunity: A Double-Edged Sword}

ILCs are distinct from conventional lymphoid lineages because they lack recombinationactivating gene (RAG)-dependent rearranged antigen-specific receptors [1-3]. ILCs are located in primary and secondary lymphoid organs in humans and mice [2]. They are mainly enriched at barrier surfaces in the body, including gut, lungs, and skin, suggesting that they are able to rapidly respond to environmental stress signals, such as inflammation [1-3]. Over the past decade, ILCs have been described as being involved in protection against pathogens, tissue remodeling, and tissue homeostasis, via the production of key factors, such as cytokines [interferon (IFN)- $\gamma$, interleukin (IL)-22, and amphiregulin (Areg); see Glossary] [2] (Figure 1 and Box 1). However, dysregulation of ILC functions now appears to contribute to the pathogenesis of several inflammatory disorders, including asthma, CD and RA [4]. In this context, ILCs may also be important players in tumor-associated inflammation. Years of research have contributed to defining the role of NK cells in controlling tumor growth and metastasis [3], but the involvement of noncytotoxic helper-like ILCs in tumors remains controversial.

In this review, we provide an overview of ILC features, as well as ILC-poiesis, highlighting the parallel between human and mouse ILC development (Boxes 1 and 2). We also discuss the ability of helper-like ILCs to convert into different ILC subsets and how these properties can impact immunity. Finally, we report recent advances regarding the involvement of helper-like ILCs in various inflammatory diseases and carcinogenesis, depicting the double-edged sword of their immune properties. A better understanding of ILC development, differentiation, and plasticity might offer new possibilities for designing specific treatments for certain diseases, aiming to induce ILC differentiation into non-inflammatory and/or antitumor subsets in vivo.

\section{General Features and Functions of ILCs in Mice and Humans}

NK Cells and ILC1s

NK cells are circulating cytotoxic cells that can lyse virus-infected normal and tumor cells [3] (Figure 1 and Box 2). By contrast, ILC1s are only weakly cytotoxic and have been reported to

\section{Highlights}

ILCs comprise NK cells, helper-like ILCs (ILC1s, ILC2s, and ILC3s) and LTi cells. ILCs are involved in defense against viruses, parasites, and bacteria, and in tissue repair and metabolic processes.

NK cells and helper-like ILCs are derived from a common ILC progenitor in both humans and mice.

The identity of mature ILCs is not fixed but is highly plastic and dependent on environmental signals.

ILCs are also involved in inflammation, several inflammatory diseases, and cancer progression. Thus, ILCs might constitute novel putative therapeutic targets for different diseases.

${ }^{1}$ Aix Marseille Univ, CNRS, INSERM, CIML, Centre d'Immunologie de Marseille-Luminy, Marseille, France

${ }^{*}$ Correspondence: narni@ciml.univ-mrs.fr (E. Narni-Mancinelli). 
be mostly resident in secondary lymphoid tissues of parabiotic mice [5,6]. However, this finding was recently challenged by a study showing that $>60 \%$ of lymph-node $(L N)$ ILC1s are present in circulation [7]. In this study, the authors took advantage of a mouse model of Kaede photoconversion [7], in which all cells of the light-exposed brachial lymph node changed their fluorescence from Kaede green to Kaede red. The presence of a substantial proportion of migratory Kaede green among Kaede red ILCs in the light-exposed lymph node a few days later demonstrated their migratory potential [7]. Similar to NK cells, ILC1s require C-C chemokine receptor 7 (CCR7) and CD62L (L-selectin) to migrate, but depend on sphingosine 1 phosphate receptor 1 (S1P1) rather than on S1P5, for egress from LNs; indeed, the S1P receptor agonist FTY720 blocked lymphocyte recirculation and caused ILC1 accumulation in LNs [7]. NK cells and ILC1s have several features in common, including IFN-y production [2]. ILC1s are the first cells to produce IFN- $\gamma$ at the site of primary infection with several mouse viruses, including the murine cytomegalovirus (MCMV), influenza virus, and Sendai virus [8]. Homolog of Blimp-1 in T cells (Hobit, Zfp683 ${ }^{-/-}$) transcription factor-deficient mice lack liver ILC1 [9], because Hobit participates in the regulation of lymphocyte tissue residency, and the control of MCMV infection is severely impaired relative to wild-type (WT) mice [8]. Moreover, IFN-y-producing ILC1s are also involved in first-line defense against intestinal infections, for instance with Toxoplasma gondii [10] and Clostridium difficile [11] in mouse models. Indeed, transfer of ILC1s has conferred substantial protection to ILC-deficient $\boldsymbol{R a g}^{-/-} \boldsymbol{\gamma} \boldsymbol{C}^{-/-}$mice infected with T. gondii [10]. Similarly, IFN- $\mathrm{Y}$-producing ILC1s among total ILCs transferred into Rag $2^{-/-} \mathrm{VC}^{-/-}$recipient mice contribute to bacterial clearance upon $C$. difficile infection [11]. At steady state, the liver, lung, intestines, and dermis contain small numbers of ILC1s in humans and mice. By contrast, ILC1 numbers are high in nonresolving Th1-like chronic inflammatory diseases, including chronic obstructive pulmonary disease (COPD) [12] and CD [13] in humans (see later).

ILC2S

ILC2s are present in most barrier tissues in humans and mice, including lungs, gut, and skin [2]. They have been described as mostly tissue resident in mouse parabionts [5,6], but the Kaede photoconversible mouse model recently revealed that a substantial fraction (40\%) of LN ILC2s are present in circulation [7]. In murine lungs, ILC2s are found close to adventitial stromal cells, a mesenchymal fibroblast-like niche that provides factors such as IL-33, and inflammatory thymic stromal lymphopoietin (TSLP), thus favoring the accumulation and activation of ILC2S in murine lungs [14] (Figure 1 and Box 2). Production of IL-13 by tissue-resident ILC2s and Th2 cell recruitment upon allergen stimulation and helminth infections in the mouse gut are responsible for a type 2 immune response known as 'weep and sweep' [15]. This response leads to protective hyperplasia of the mucus-secreting goblet cells during mouse infections with the helminth Nippostrongylus brasiliensis [16] and the nematode Heligmosomoides polygyrus [17], relative to controls. In the case of viral infections, such as with influenza virus, or when using the dextran sodium sulfate (DSS) model of intestinal colitis, once the inflammation is resolved, ILC2s may participate in tissue repair by producing Areg [18,19]. Indeed, anti-Thy1.2-mediated depletion of ILCs in the lungs of influenza virus-infected mice led to epithelial degeneration and necrosis, as well as decreased Areg mRNA expression relative to isotype control-injected mice [19]. In addition, there was increased numbers of Areg-expressing ILC2s in response to DSSinduced intestinal injury, which correlated with improved disease severity in mice [18]. Recently, using a mouse model of ILC2 deficiency caused by the lack of a transcription factor, promyelocytic leukemia zinc finger protein (PLZF, Zbtb16; Zbtb16 ${ }^{-/-}$mice), a study showed that these mice were more susceptible to Staphylococcus aureus infection relative to WT mice [20]. ILC2s were reported to protect mice from lethal bacteremia by secreting type 2 cytokines, promoting eosinophilia and preventing a lethal neutrophilic response [20]. Lastly, decreased numbers of ILC2s in human and mouse white adipose tissue have been associated with obesity

\section{Glossary}

Adipose tissue: contains an energystoring compartment called white adipose tissue (WAT), a thermogenic one named brown adipose tissues (BAT), and some thermogenic BAT-like adipocytes (brite/beige) that are interspersed within WAT.

Amphiregulin (Areg): crucial growth factor supporting the growth and survival of epithelial cells.

Cholangiocytes: epithelial cells of the bile duct.

Dextran sodium sulfate (DSS) model: inflammation model; induces damage to the epithelial monolayer lining the colon, mimicking colitis.

Goblet cells: intestinal epithelial cells specialized in mucus secretion. Helper-like ILCs: ILC1s, ILC2s, and ILC3s considered to be noncytotoxic innate counterparts of adaptive helper $T$ cells.

ILC-deficient Rag2 $^{-1-} \boldsymbol{r c}^{-/-}$mice: the recombination activation gene Rag2 is required by $T$ and $B$ lymphocytes for $V$ (D) $J$ recombination, whereas the common y chain of IL-2R (II2rg ${ }^{-1-}$ called $\mathrm{YC}^{-/-}$here) is part of many cytokine receptors, including IL-2, IL-4, IL-7, IL-9, and IL-15. As such, these mice lack $T$ and B cells and ILCs.

Induced bronchus-associated lymphoid tissue: ectopic lymphoid tissue adjacent to major airways; formed upon inflammation or infection. It initiates loca immune responses and maintains memory cells in the lungs. Intermediate ILC1s (IntILC1s): cell subset resulting from the conversion of NK cells into ILC1-like cells; observed in the microenvironment of MCA-induced fibrosarcomas and also upon Toxoplasma gondii infection in mice; produce large amounts of TNF- $\alpha$ that might promote tumor growth and facilitate metastasis. Their role in infection remains undetermined. Lgr5 ${ }^{\text {CreERT2 }}$ II22ra $1^{\text {f/f }}$ mice: model that allows for inducible II22 receptor inactivation in crypt-based columnar stem cells via tamoxifen-inducible Cre activity.

Lymphoid tissue inducer cells (LTis): have a key role in the development of second lymphoid organs (e.g., lymph nodes and Peyer's patches).

Mouse model of Kaede photoconversion: Kaede green fluorescent protein is irreversible photoconverted to red after exposure to 
[21]. ILC2s contribute to regulating the metabolic homeostasis of lipids and glucose, given that transfer of IL-33-activated ILC2s to obese mice (high-fat diet induced) was associated with metabolic 'beiging', a process known to increase caloric expenditure by reducing lipids stored within adipose tissue [22]. Taking together, these studies indicate that ILC2s, by providing a type 2 immune response, are important players in parasitic infections and tissue repair, and can also participate in metabolic processes. From another angle, ILC2s are considered to be major inducers of Th2-mediated inflammatory diseases in the airways, intestine, and skin in both humans and mice (see later) [4,23].

ILC3S

ILC3s are particularly abundant in human and mouse mucosae, particularly in the gut [2], and are largely resident, because only $20 \%$ of green Kaede cells were recovered from light-exposed LN a week after photoconversion in mice [7]. ILC3s have also been implicated in the confinement of intestinal commensals [24]; indeed, loss of ILC3-derived IL-22 in either transcription factor Rorc-deficient mice, or upon ILC3 depletion (using an anti-Thy1.2 antibody in Rag1-deficient mice), resulted in the translocation of commensal bacteria to peripheral organs, such as the spleen and liver [24]. ILC3s also provide an innate immune response to extracellular bacteria [24] (Figure 1 and Box 2). Recently, lung ILC3s were reported to contribute to early protection against Mycobacterium tuberculosis in mice, by generating lymphoid follicles within granulomas associated with immune protection against this bacterium [25]. Indeed, ILC3 functional deficiency of the $\boldsymbol{R o r c}^{\mathrm{Cre}} \boldsymbol{A} \boldsymbol{h r ^ { f / f }}$ mouse model caused increased bacterial burden and decreased formation of induced bronchus-associated lymphoid tissue (BALT) structures relative to WT mice [25]. Mouse ILC3s also maintained intestinal homeostasis by producing IL-22; injection of either IL-22neutralizing or ILC-depleting monoclonal antibodies (mAbs) to Rag1-deficient mice resulted in the dissemination of live commensal A/caligenes bacteria and the induction of systemic inflammation [26]. ILC3s have also been shown to limit the pathological activity of Th17 cells in response to commensal bacteria (colonic extract) via $\mathrm{MHC}$-II-dependent interactions, resulting in the negative

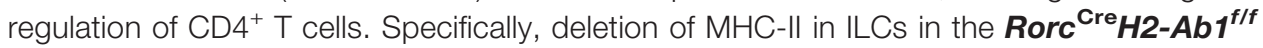
mouse model resulted in increased frequencies of IL-17-producing gut CD4 ${ }^{+} \mathrm{T}$ cells and increased serum IgG against commensal bacteria relative to $\mathrm{H} 2-A b 1^{\mathrm{f} / f}$ mice [27]. Moreover, recent work using a mouse model of colitis-associated colon cancer [procarcinogen azoxymethane (AOM) treatment, coupled with DSS-induced inflammation], indicated that IL-22, mainly derived from ILC3s, protected intestinal stem cells against genotoxic stress in the mice [28]. In this study, the authors showed that the absence of IL-22 signaling into intestinal epithelial stem cells, which lack the IL-22 receptor (Lgr5 ${ }^{\text {CreERT2}} \mathbf{I I 2 2 r a 1 ^ { f / f }}$ mice), impaired DNA damage-induced apoptosis of colon stem cells and resulted in higher numbers of tumors following DSS-induced inflammation relative to control mice [28]. ILC3s also produce IL-17 in response to IL-23, IL-1 $\beta$, and IL-6, recruiting neutrophils and macrophages to induce inflammation in mice [24]. Lastly, both mouse and human ILC3s can produce GM-CSF, which is involved in modulating proper regulatory $\mathbf{T}$ cell (Treg) priming and immune tolerance towards commensals in the gut [29]. Indeed, Rorc-deficient mice exhibit a defect in GM-CSF production by ILC3s and LTis in the gut, resulting in altered mononuclear phagocyte effector functions and decreased production of Tregs relative to controls [29]. In this study, using mice sensitized to ovalbumin (OVA) (oral gavage) and stimulated subcutaneously by OVA as a model for the skin delayed type hypersensitivity (DTH) response, the defect in ILC3 GM-CSF and Treg production resulted in impaired oral tolerance to food antigen in the affected mice [29]. Together, these studies demonstrate that ILC3s are involved in extracellular bacterial defense and are crucial for regulating immune tolerance towards commensals, in addition to ensuring the confinement of these microbes. Thus, dysregulated ILC3 functions would likely contribute to the development of inflammatory disorders in mucosae-associated tissues (see later). ultraviolet light, allowing the study of cell migration in vivo.

Ncr1 ${ }^{\text {Cre }}$ Rosa26 $6^{\text {ffYFP }}$ fate-mapping mouse model: mice with NKp46expressing cells and cells that have expressed NKp46 but do not express it anymore (fate-mapped; YFP ${ }^{+}$). NGS mice: strain of immunodeficient laboratory mice used for humanized mouse models. These mice lack mature T cells, B cells, and NK cells. Parabiotic mice: two mice surgically joined by skin flaps, which develop a single shared bloodstream by chimerism of blood vessels and blood flow. Regulatory T cells (Tregs): T cell subset, formerly known as suppressor $T$ cells, that modulate the immune system; needed to maintain tolerance to selfantigens and to prevent autoimmunity. RNA velocity: bioinformatics algorithm defining the time derivative of the gene expression state estimated by the discrimination between unspliced and spliced mRNAs; may predict the future state of an individual cell, helping to analyze developmental cellular lineages and dynamics, such as plasticity. Rora $^{\text {sg/sg }}$ mouse model: mice harboring a spontaneous deletion in Rora leading to the loss of Rora activity. Rorc ${ }^{\text {Cre }} \boldsymbol{A} \boldsymbol{h r}^{\mathrm{f} / \mathrm{f}}$ mice: the aryl hydrocarbon receptor (Ahr) gene, encoding a ligand-dependent transcription factor important for ILC3 function and survival, is deleted in Rorcexpressing cells. As such, these mice lack functional ILC3s.

$\operatorname{Rorc}^{\mathrm{Cre}} \boldsymbol{H 2}-\boldsymbol{A b} \mathbf{1}^{\mathbf{f} / \mathrm{f}}$ mice: the MHC-II beta chain $\mathrm{H} 2-A b 1$ is deleted in Rorytexpressing cells. As such, these mice lack MHC-II in ILC3s.

Skin delayed type hypersensitivity (DTH) response: develops 1-3 days after exposure to an antigen recognized as foreign. This response relies mainly on $T$ cells rather than on $B$ cells and can lead to tissue damage in the presensitized host.

Sphingosine 1 phosphate receptor 1/5 (S1P1 and S1P5): G-proteincoupled receptors; bind the bioactive lipid S1P involved in lymphocyte migration. S1P1 controls egress of $T$ and $B$ cells from secondary lymphoid organs. S1P5 is expressed in NK cells and is required for their egress from bone marrow and lymph nodes as well as for their recruitment to inflamed tissues.

ST2, II1r1: member of the IL-1 receptor family. The ligand for ST2 is the cytokine 


\section{Recent Advances in ILC Development}

Recent work identified circulating human ILC precursors in the blood and secondary organs [30]. However, similar ILC precursors remain elusive in the mouse. The identification of ILC progenitors is of interest because it would make it possible to promote the development of these cells in curative approaches to treat various diseases. For example, the promotion of ILC2 development in adipose tissues might constitute a novel approach to promote systemic metabolism in obese individuals [21]. Moreover, current studies of ILC-poiesis have revealed greater similarities between the development of NK cells and ILCs than previously thought.

\section{Murine ILC Development}

ILCs are derived from the common lymphoid progenitor (CLP), which can give rise to all lymphocyte subsets, including ILCs, and B and T cells (Figure 2) [31]. Nfil3 is required for the generation of the ILC precursor, because mouse models in which this transcription factor was knocked out lacked mature ILCs, including NK cells [32]. Recently, via whole-genome analysis of in vivoderived cells, CXCR6 ${ }^{+} \alpha_{4} \beta_{7}^{+} I L-7 R^{+} \alpha$-lymphoid precursor ( $\alpha \mathrm{LP}$ ) cells were found to differentiate from the murine CLP [33]. A combination of genetic tracing and phenotypic, functional, and bioinformatic approaches showed the generation of TCf7 fate-mapped Lin ${ }^{-T h y} 1.2^{-} \alpha_{4} \beta_{7}^{+} I L-7 R^{-}$early innate lymphoid progenitors (EILCPS) downstream from the murine CLP [34]. Given the drastic decrease in the number of aLP in transcription factor Tcf7-deficient mice relative to WT mice [33], it is likely that aLP constitutes an intermediate between CLP and EILCP, although this remains to be further tested. Early-stage EILCPs (specified as EILP1s), can give rise to ILCs and dendritic cells (DC), and differentiate into committed EILPs (cEILCP) upon TCF1 expression [35]. Moreover, cEILCP can give rise to all ILCs and NK cells, because $T c f 7^{-1-}$ mice lack all ILC populations, and their expression is required for CEILCP generation and/or maintenance [36]. Using an $/ d 2^{\text {GFP }}$ reporter mouse, one study showed that ILCs and NK cells differentiated from EILCPs, and branched via Id2-dependent $\mathrm{Lin}^{-} \mathrm{Id} 2^{+} \mathrm{IL}-7 \mathrm{R}^{+} \mathrm{IL}-2 \mathrm{R \alpha ^{- }} \mathrm{\alpha}_{4} \beta_{7}^{+}$common helper-like ILC progenitors (CHILP), and Id2-independent NK1.1 $1^{-} \mathrm{IL}-2 \mathrm{R} \beta^{+} \mathrm{NK}$ progenitors (NKP) $[9,34,36-38]$. CHILPs can give rise to helper-like ILCs (ILC1s, ILC2s, and ILC3s) and LTis but not to NK cells [10]. Conversely, NKPs give rise to NK cells, but not to ILCs [39]. CHILPs can be separated into subsets based on Zbtb16 (PLZF) expression. The Zbtb16 ${ }^{+}$subset, or ILC precursor (ILCP), can no longer produce LTi cells, whereas this capacity is retained by the Zbtb $16^{-}$subset $[10,40]$. Zbtb $16^{+}$CHILPs also display cell-surface expression of programmed cell death 1 (PD-1), a marker of early progenitors and of activated ILCs in mice [41]. However, two groups recently revisited this developmental model, using the $/ d 2^{\mathrm{RFP}} Z$ btb $16^{\mathrm{CreGFP}} \mathrm{Bc} / 11 b^{\text {tdTomato }}$ and

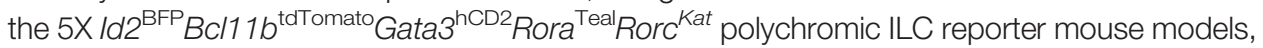
to isolate multipotent ILCPs and NKPs, respectively, from Id $2^{+}$progenitors $[42,43]$. Specifically, essential transcriptions factors involved in ILC differentiation were tagged by fluorescent proteins in these mice: Bc/11b, Gata3, and Rora for ILC2 and Rorc for ILC3. Id2 expression was evident because of the brightness of the fluorescent markers and detected in ILCPs and NKPs [42,43], in contrast to that observed in the $/ d 2^{\mathrm{GFP}}$ mouse model [10]. The authors showed that $/ d 2^{+} \mathrm{Zbtb} 16^{-}$ cells and $/ d 2^{+} B c / 11 b^{-n i p p o /+}$ Gata3 ${ }^{-}$cells could differentiate into all ILCs, including NK cells [43]. Thus, according to this revised model, committed NKPs may lie downstream of a common ILC precursor (CILCP) capable of giving rise to both cytotoxic conventional NK cells and helper-like ILCs (Figure 2) [42,43]. The earliest ILC3 progenitor was contained within a small fraction of $/ d 2^{+} B c / 11 b^{+}$Gata $^{\text {low }}$ cells expressing Rorc ${ }^{\text {Kat }}$ [42]. Mouse $/ d 2^{+} Z$ Zbtb $16^{+}$ILCPs gave rise to conventional NK cells both in vitro and in vivo, and to ILC1s and ILC2s, but comparatively exhibited a reduced potential for differentiating into CCR6 ${ }^{+}$ILC3S [42]. In addition, ILC2S could differentiate from $/ d 2^{+} B c / 11 b^{+} Z b t b 16^{-}$and Zbtb $16^{+}$precursors. Indeed, ILC2Ps were predominant among the $\mathrm{Id} 2^{\mathrm{BFP}+}$ bone marrow cells, and Id $2^{\mathrm{BFP}+} \mathrm{Bcl} 11 \mathrm{~b}^{\text {tdTomato+ }} \mathrm{Gata} 3^{\text {hCD2hi }}$ cells differentiated exclusively into ILC2 progeny [42]. Thus, constitutive Id2 expression is required
IL-33; therefore, ST2 is also known as IL-33R.

Tertiary lymphoid structures: ectopic lymphoid organs usually found at chronic inflammation sites, exhibiting all characteristics of typical lymph nodes. Their presence can be beneficial or detrimental depending on the causes of inflammation.

Th1-like: related to Th1-type cytokines classically associated with Th1 immune; IFN-y is the main Th1 cytokine.

Th17 cells: subset of proinflammatory

Thelper cells developmentally distinct from Th1 and Th2 lineages producing cytokines including IL-17A, IL-17F, IL21, and IL-22.

Thymic stromal lymphopoietin (TSLP): cytokine signaling through a heterodimeric receptor complex comprising TSLP receptor and IL-7R TSLP promotes Th2 responses associated with immunity in various inflammatory diseases.

Type 2 immune response: characterized by antibody-mediated immunity and release of cytokines (including IL-4, IL5, and IL-13); needed for extracellular pathogen control and allergy.

Weep and sweep: during helminth infections, IL-4 and IL-13 cytokines increase intestinal mucus secretion (weep) and muscle contractility (sweep), favoring parasite expulsion rather than killing. 
(A)

(B)

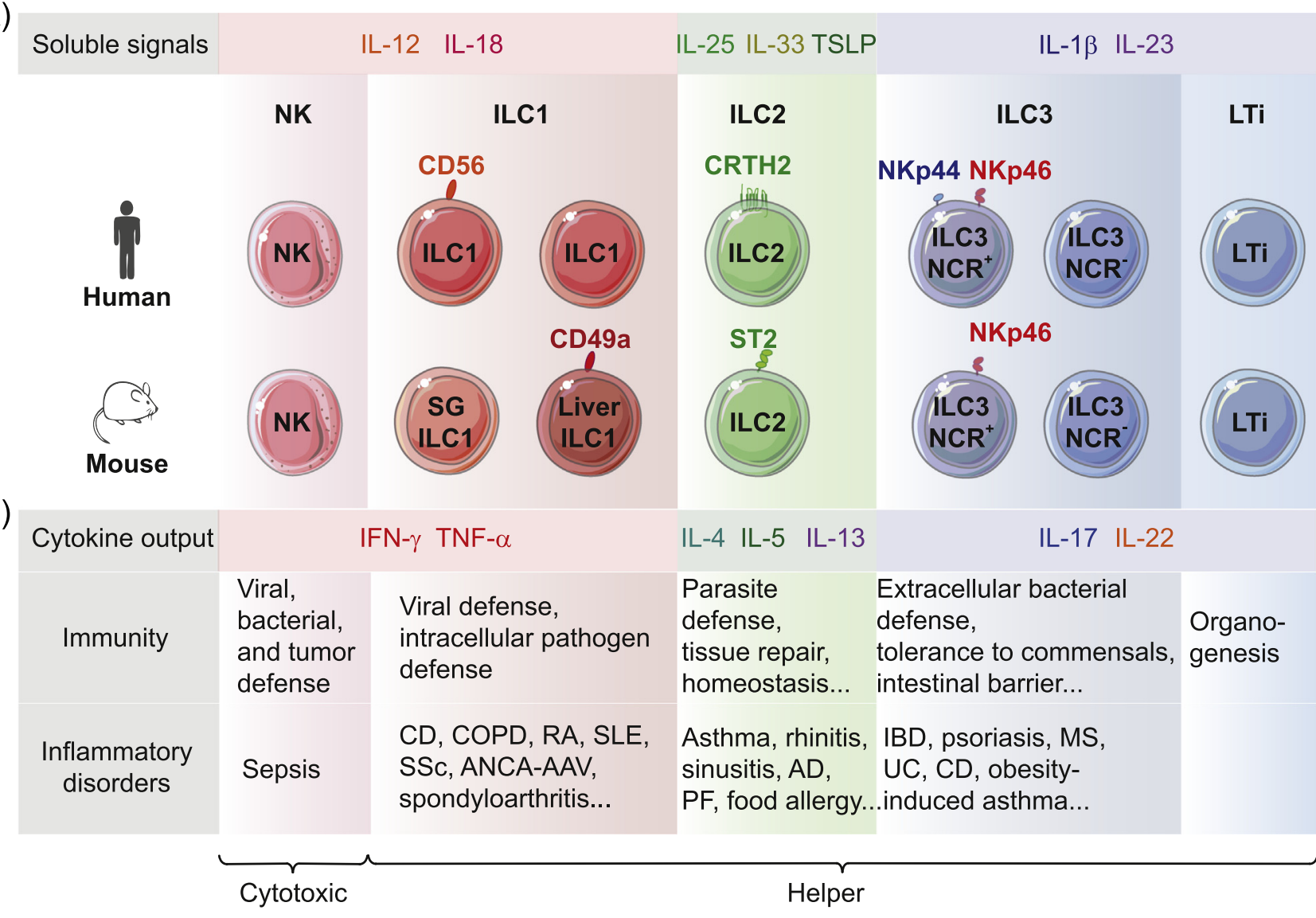

Trends in Immunology

Figure 1. General Features of Innate Lymphoid Cells (ILCs). (A) Different ILC subsets described in humans and mice. Natural killer (NK) cells and ILC1s are sensitive to interleukin (IL)-12 and IL-18 signals and produce interferon-gamma (IFN- - ) and tumor necrosis factor-alpha (TNF- $\alpha$ ), whereas ILC2s respond to IL-25, IL-33, and thymic stromal lymphopoietin (TSLP) soluble molecules by producing IL-4, IL-5, and IL-13 Th2 cytokines [2]. ILC3s and lymphoid tissue inducer cells (LTis) can sense IL-1 $\beta$ and IL23, activating the production of IL-17 and IL-23 [2]. NK cells are cytotoxic, whereas helper-like ILCs are not. Helper-like ILC1s can induce cell death through the TNF-related apoptosis-inducing ligand (TRAIL) pathway [2]. (B) Overview of the pros and cons of ILCs in immunity. Abbreviations: AD, atopic dermatitis; ANCA-AAV, acute-phase antineutrophil cytoplasmic antibody-associated vasculitis; CD, Crohn's disease; COPD, chronic obstructive pulmonary disease; CRC, colorectal cancer; EoE, eosinophilic esophagitis disorder; IBD, inflammatory bowel disease; MS, multiple sclerosis; NSCLC, nonsmall cell lung cancer; PF, pulmonary fibrosis; RA, rheumatoid arthritis; SLE, systemic lupus erythematosus; SSc, systemic sclerosis.

for ILC development, whereas Zbtb16 is not [33]. Moreover, LTis are thought to be derived from CHILPs [10], but the precise branchpoint needs to be reconsidered in light of recent evidence regarding murine ILC development, as discussed earlier [42,43] (Figure 2).

Human ILC Development

Human ILCs differentiate similar to mouse ILCs, beginning with $\mathrm{Lin}^{-} \mathrm{CD} 34^{+} \mathrm{CD} 45 \mathrm{RA}^{+} \mathrm{CD} 10^{+} \mathrm{KIT}{ }^{-}$ CLPs [44] (Figure 2). NK developmental intermediates are thought to differentiate from this progenitor, at least in secondary lymphoid tissues, such as the tonsils and lymph nodes [45]. The most immature precursors are the early tonsillar progenitors (EToP), with a $\mathrm{Lin}^{-} \mathrm{CD} 34^{+} \mathrm{CD} 10^{+} \mathrm{KIT}^{-}$ (EToP1) or $\mathrm{Lin}^{-} \mathrm{CD} 34^{+} \mathrm{CD} 10^{-} \mathrm{KIT}^{+}$(EToP2) phenotype $[46,47]$. Both of these populations are multipotent and can also give rise to both T cells and DCs in vitro [46]. EToP2 is heterogeneous in terms of ST2 (IL-33R, I/1r1) expression; the EToP2 ST2- population is multipotent but has a greater ILC-generating potential than EToP1 cells, giving rise to a larger proportion of ILCs than to T cells or DCs in vitro [46]. By contrast, EToP2 ST2 ${ }^{+}$cells give rise exclusively to ILCs both 
Box 1. Nomenclature of ILCS

In 2013, ILCs were classified into three groups in human and mouse on the basis of their cytokine secretion profiles and the transcription factors expressed during their development [1]. Group 1 ILCs comprised cytotoxic NK cells and noncytotoxic ILC1s, both of which are dependent on the T-box transcription factor 21 (TBET) for their development and function, and produce IFN-y [1]. NK cells and ILC1s differ in terms of their expression status for a transcription factor, Eomesodermin (EOMES). Group 2 contains a single subset, ILC2s, which are dependent on the transcription factors RAR-related orphan receptor $\alpha$ (RORa) and GATA-binding protein 3 (GATA3), and produce predominantly type 2 cytokines (IL-13 and IL-5) [1]. Group 3 ILCs include three subsets: LTi, the natural cytotoxicity receptor (NCR) ILC3s, and the NCR ILC3s, which produce IL-17 and/or IL-22 and are dependent on the transcription factor RORyt [1]. Extensive research in ILC-poiesis in recent years has led to refinement of the classification of the ILC family. Five subsets have now been defined on the basis of developmental trajectories: NK cells, three subsets of helper-like ILCs (ILC1s, ILC2s, ILC3s), and LTi cells [2].

in vitro and in vivo when isolated from pediatric tonsils and injected into immunodeficient NGS mice [47], and cannot generate non-ILC cells. Thus, these cells are the earliest committed human CILCP identified to date [47]. A $\mathrm{Lin}^{-} \mathrm{CD} 34^{-} \mathrm{CD} 7^{+} \mathrm{IL}-7 \mathrm{R}^{+} \mathrm{KIT} \mathrm{T}^{+}$ILC progenitor (ILCP) with a restricted potential for ILC generation was recently identified in cord blood, fetal liver, peripheral blood, and other human tissues [30]. A more restricted progenitor has been identified downstream of this ILCP: the CD34 ${ }^{-} \mathrm{KIT}{ }^{+} \mathrm{CD} 56^{+}$restricted ILCP (rILCP [48]), which gives rise to NK cells, ILC1s, and ILC3s, but not to ILC2s [49] (Figure 2).

Thus, several different ILC precursors have been identified, and are probably capable of giving rise to specific helper-like ILCs in tissues. Nevertheless, the identity of mature helper-like ILCs remains rather plastic.

\section{Plasticity of Mature ILCs}

Several studies have shown that the phenotype and functional capacities of ILC subsets can change (Figure 3). Such conversion has been observed for mouse CCR6 ${ }^{-} \mathrm{NKp} 46^{-}$gut ILC3s,

\section{Box 2. Phenotypes of Mouse and Human ILCs}

Mouse NK cells express NKp46 and Eomes, but lack CD3, whereas human NK cells have a CD3-CD56 ${ }^{+} \mathrm{CD} 94^{+}$phenotype [2]. Human helper-like ILCs exclude cells expressing CD3 (T cells), CD19 (B cells), CD94 (NK cells), and CD14 (myeloid cells), but include those expressing IL-7R. ILC1s can be subdivided into two subpopulations. The first, in tonsils and the intestinal intraepithelial space, expresses CD56 and CD103, produces IFN- $y$, and contains granules with perforin and granzyme B [10], thus closely resembling NK cells. The other, in the human lamina propria, expresses IL-7R, CD161, and TBET, but not CD56, CD94, granzyme B, perforin, or EOMES [13], and produce IFN- $y$ in response to IL-12 and IL-18. The ILC1 phenotype remains unclear. Mouse liver and human intraepithelial ILC1s preferentially express TRAIL and CD49a [2]. They also express HOBIT and BLIMP1, which appear to regulate the expression of tissue-residency molecules $[9,123]$. Other ILC1s have phenotypic markers in common with ILC3s and NK cells in some organs, including NK1.1 in mice, NCRs (e.g., NKp44) in humans, and NKp46 in both humans and mice [2]. The expression of both IL-7R and CD200R distinguishes ILC1s from NK cells in mice. IL-7R is not a human ILC marker because it is not expressed by some CD56 bright CD16 ${ }^{-}$NK cells in peripheral blood. By contrast, ILC1s do not express NKp80, a marker of NK cells [124]. It is almost impossible to isolate ILC1s from human tissues without contamination with other lineages [125]. However, ILC1s can be derived from NK cells in the tumor microenvironment in MCA-induced mouse fibrosarcomas [65].

Mouse and human ILC2s are phenotypically similar and express IL-7R, ST2 (IL-33R or IL-1R-like 1), IL-2Ry (CD132), IL2Ra (CD25), IL-17RB, and TSLPR [2], at least at steady state [126]. Mouse ILC2s also express KLRG1, ICOS, and SCA1 [23], whereas human ILC2s express the chemoattractant receptor-homologous molecule expressed on Th2 cells (CRTH2), CD161 (C-type lectin receptor), and cysteinyl leukotriene receptor 1 (CysLT1R) $[83,127,128]$. Surface expression of these markers depends on ILC2 activation and appears to be variable over time; it also differs between tissue compartments [126]. Typical ILC2s produce type 2 cytokines (IL-13, IL-4, and IL-5) and respond to Th2-proinflammatory IL-25, IL-33, and TSLP cytokines (see Figure 1 in the main text) [2].

ILC3 development and function depend on Rorc in mice [2], whereas patients with RORC deficiencies lack the IL-17Aproducing ILC3 subset, but not the IL-22-producing subset [30]. ILC3s can be subdivided in two subsets, NCR ${ }^{-}$and $\mathrm{NCR}^{+}$ILC3s, on the basis of cell-surface NKp44 (in humans) or NKp46 (in mice) expression [2]. 


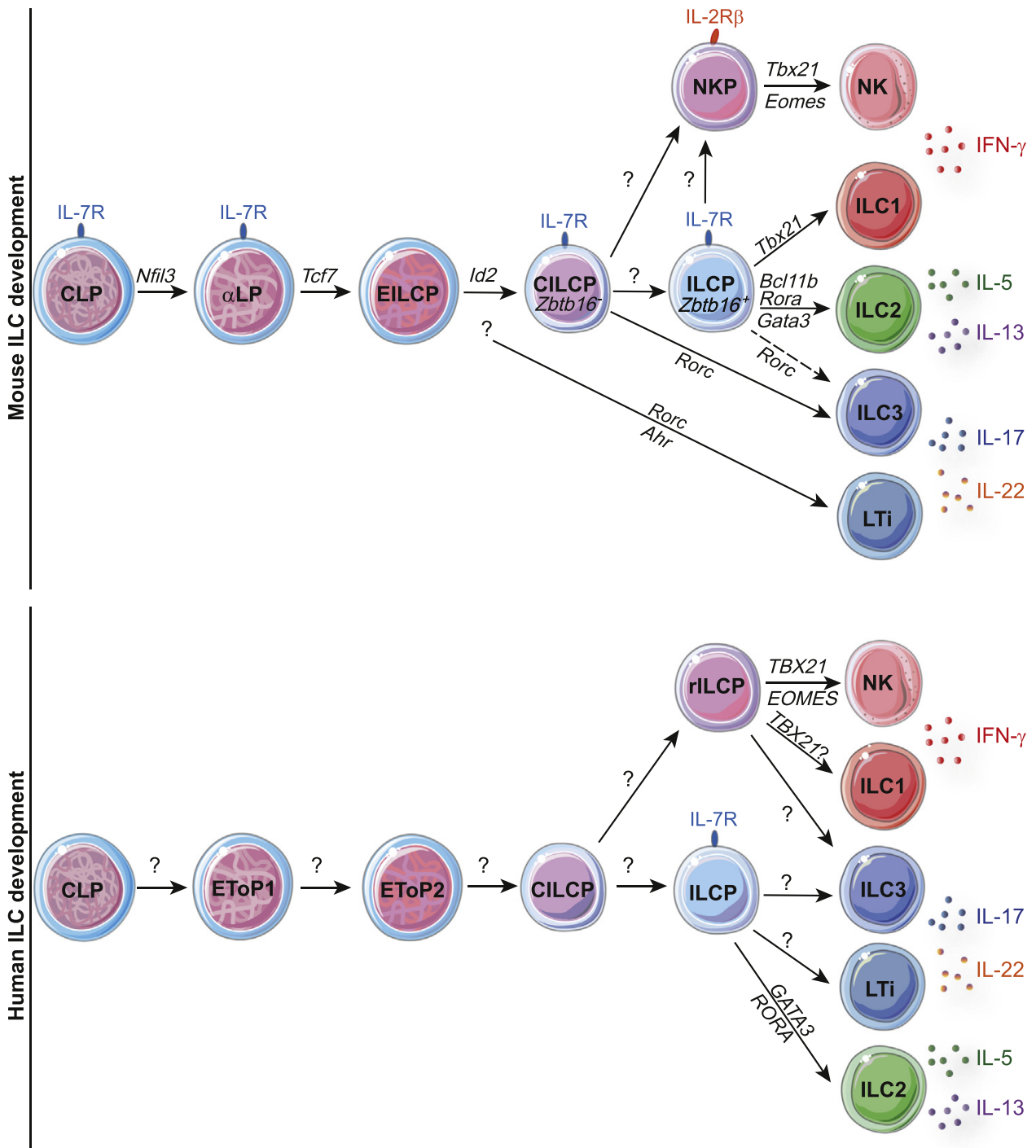

Trends in Immunology

Figure 2. Mouse and Human Innate Lymphoid Cell (ILC) Development. The different steps of mouse and human ILC development are shown. Abbreviations: aLP, a-lymphoid precursor; CILCP, common ILC progenitor; CLP, common lymphoid progenitor; EILCP, early innate lymphoid cell progenitor; EToP, early tonsillar progenitors; ILCP, ILC precursor; NK, natural killer; NKP, NK cell precursor; rlLCP, restricted ILC precursor.

which can convert into NK1.1 $1^{+} \mathrm{NKp} 46^{+}$IFN-y-producing ILC1s [50], and for human IL-22producing ILC3s, which can convert into IFN- $y$-producing ILC1-like cells, when cultured with IL-23 and IL-1 $\beta$ [13,51]. The Ncr $^{\text {Cre }}{ }^{\text {Rosa2 }}$ f $^{\text {ffYFP }}$ mouse fate-mapping model [52] revealed the phenotypic plasticity of NKp46 expression in mucosa-associated NKp46 ${ }^{+}$ILC3 subsets [53]. Fate-mapped eYFP ${ }^{+} \mathrm{NKp} 46^{-}$ILC3s that previously expressed NKp46 were identified and their conversion may be dependent on TGF- $\beta$ [53]. In addition, human and mouse cytotoxic NK cells can acquire ILC1-like features in tumors in response to TGF- $\beta$ signaling [54]. Indeed, Smad4-deficient mouse NK cells exhibit a ILC1-like phenotype, such as surface expression of CD49a for mouse cells, and CD103 and CD9 for human cells, as well as decreased antitumor 


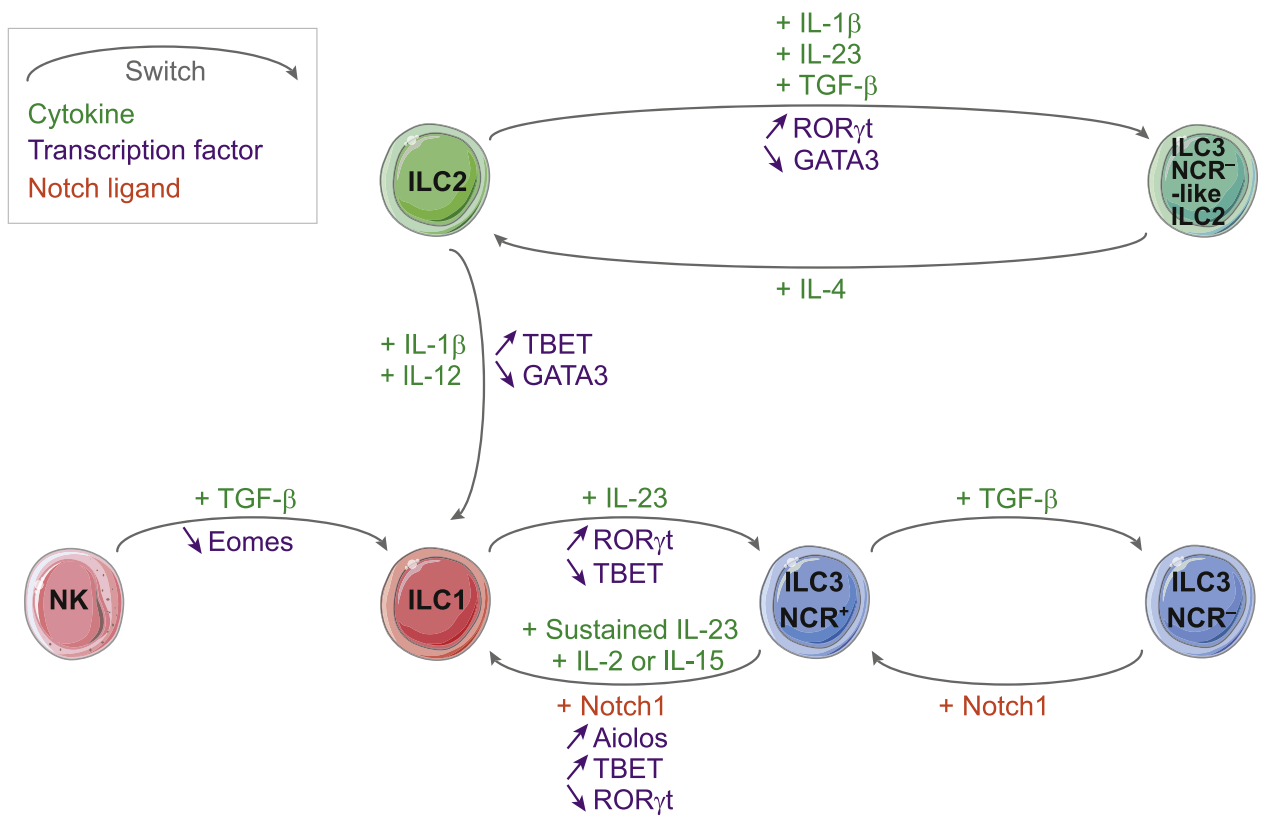

Trends in Immunology

Figure 3. Innate Lymphoid Cell (ILC) Plasticity. Specific subsets of ILCs can switch into another subset depending on the cytokines, transcription factors and $\mathrm{NOTCH}$ ligands present in their environment. This phenomenon is called 'ILC plasticity'. Abbreviations: Eomes, eomesodermin; GATA3, GATA-binding protein 3; IL, interleukin; NCR, natural cytotoxicity receptor; ROR, RAR-related orphan receptor; TBET, T-box transcription factor 21; TGF, transforming growth factor

properties [54]. Expression of Smad4 appears to control the TGF- $\beta$ imprinting of mouse NK cells [54]. Single-cell RNA sequencing (scRNAseq) of human tonsil and intestine ILC3s and ILC1s and bioinformatics analysis (RNA velocity) allowed the recent identification of a cluster of cells representing a spectrum of cell conversion between ILC3s and ILC1s, with a pronounced directionality toward ILC1s [55]. Cell transfers into humanized mouse recipients demonstrated the in vivo conversion of ILC3s into ILC1s, which occurred preferentially in tissues expressing TGF$\beta$, such as the spleen [55]. Moreover, transcriptional repression by mouse transcription factors Aiolos and T-bet was required to regulate active elements in ILC3s, such as IL22 and Rorc, and to favor their conversion into ILC1s [55]. Indeed, Aiolos bound near IL22 and Rorc in the ILC3-like mouse cell line MNK3 transduced by Aiolos and T-bet but not near to Ifng or Eomesodermin (Eomes), suggesting selective regulation [55]. Further studies are required to address the possible conversion of ILC1s into ILC3s, although human ILC1s can convert in vitro into ILC3-producing IL-17 when co-cultured with pulmonary squamous cell carcinomas producing IL-23 [56]. Overall, the biological impact of ILC3 plasticity in vivo remain unresolved.

Recent studies reported the conversion of human and mouse ILC2s into IFN- $\gamma$-producing ILC1s in vitro when cultured with IL-12, and IL-1 $\beta$ primes ILC2s for IL-12-induced conversion into ILC1like cells $[12,57]$. In vivo, transferred GFP' ILC2s in mice converted into ILC1-like cells producing IFN-y upon influenza virus infection [58]. Furthermore, ILC2s responding to IL-25 were reported to arise in mouse lungs from resting intestinal ILC2s in response to N. brasiliensis infection [59]. These cells can migrate into the bloodstream to effector sites, to protect tissues [59]. In contrast to natural ILC2s, IL-25-elicited ILC2s express RORyt and IL-17, downregulate ST2, and upregulate KLRG1; they may also constitute a different subset or different activation state of ILC2s [59]. At steady state, ILC2s contain higher levels of II10 mRNA than other ILCs [60] and can 
also develop into a subset of IL-10-producing regulatory ILCs in the lungs of mice treated with IL33, or with the protease-allergen papain [61], limiting inflammation and eosinophil recruitment to the lungs. Human ILC2s stimulated with IL-1 $\beta$, IL-23, and TGF- $\beta$ produce IL-17 and resemble NKp44- ILC3s [62,63], apparently without the need for NOTCH1 signaling [63]. These $\mathrm{KIT}^{+-}$ $\mathrm{RORyt}^{+} \mathrm{CRTH} 2^{-}$ILC3-like cells accumulate in the skin lesions of patients with psoriasis and can constitute a major source of pathogenic IL-17 [62]. These ILC3-like cells have also been found to transdifferentiate in the nasal polyps of patients with cystic fibrosis and to convert back to CRTH2+ ILC2s when cultured with IL-4 [63].

Mouse NK cells downregulate Eomes and can resemble ILC1s not only upon in vitro culture with TGF- $\beta$, as discussed earlier [54], but also within the tumor microenvironment upon TGF- $\beta$ signaling $[64,65]$. One study reported an increase in the population of CD49b ${ }^{+} \mathrm{CD} 49 \mathrm{a}^{+}$cells, called 'intermediate ILC1s' (intILC1s), in methylcholanthrene (MCA)-induced tumors, and experimental RM-1 and B16F10 lung metastases, at the expense of NK cells. The authors showed that TGF- $\beta$ receptor-deficient NK cells transferred intratumorally gave rise to decreased numbers of intILC1s relative to the transfer of WT NK cells [65]. Such conversion of NK cells into ILC1-like cells was also recently observed in the context of $T$. gondii infection in mice [66]. In this study, the authors showed that ILC1-like cells exhibited a unique gene expression and epigenetic profile. In addition, these cells persisted independently of parasite replication, suggesting a permanent conversion of NK cells into ILC1-like cells [66]. The role of these cells during infection remains to be investigated.

The functional impact of ILC plasticity is unclear, but it is probably important for rapid immune responses to various infections, bypassing differentiation from progenitors. However, it is reasonable to speculate that these types of conversion might also lead to, or contribute to certain inflammatory states.

\title{
Helper-like ILCs in Inflammatory Diseases
}

Our understanding of the purpose of helper-like ILCs remains incomplete (Box 3), but these cells clearly participate in the development of inflammatory diseases in several conditions (Figure 1).

\begin{abstract}
Box 3. Biological Role of Helper-like ILCs
Helper-like ILCs remained unidentified for many years, and their biological role remained unclear. Indeed, helper-like ILCs do not appear to be essential for host survival in individuals with an intact adaptive immune system [122]. In most mouse models, helper-like ILC deficiencies result only in delayed pathogen clearance or minor alterations to adaptive immunity, with little impact on outcome [2]. ILCs also appear to be dispensable for protective immunity in humans [122]. Patients with severe combined immunodeficiency (SCID) undergoing hematopoietic stem cell transplantation (HSCT) without prior chemoablation conditioning do not reconstitute helper-like ILCs after transplantation. However, in the long term, the frequency of infections complications is no higher in these patients than in those with effective ILC constitution [122]. This study focused on blood ILCs rather than on tissue-resident helper-like ILCs and the cohort was small, with too short a follow-up period to analyze cancer development correctly. Nevertheless, these results have raised questions about the role of helper-like ILCs. The simplest explanation appears to be that helper-like ILCs were required long ago to protect vertebrates against a group of pathogens that no longer exists, and that they have been conserved during evolution because they confer a slight advantage. For example, helper-like ILCs produce three canonical cytokines, Th1 (IFN-y), Th2 (IL-5) and IL-17, which have been strongly conserved during evolution [129]. Our lack of knowledge of the role of these cells may also be due to an absence of appropriate tools, including mouse models or specific antibodies, for the selective depletion of ILCs in vivo, which has impaired our ability to discover the nonredundant functions of these cells in health and disease.
\end{abstract}

A role of helper-like ILCs in sensing the immunological state of tissues was recently suggested [130]. Given the plasticity of helper-like ILCs, tissue-resident ILCs probably adapt dynamically to a given stimulus to reach the appropriate state for shaping the tissue landscape [103]. Extensive research in the field and the generation of new tools, especially mouse models for the specific targeting of helper-like ILCs, should shed light on the biological role of these cells. 
ILC1S

The major cytokine produced by ILC1s is IFN- $\gamma$, which has been implicated in many inflammatory diseases and can be targeted clinically (Table 1). Relative to healthy individuals, ILC1 numbers can be increased in bronchial samples and peripheral blood from patients with chronic obstructive pulmonary disease (COPD) [58], inflamed intestinal samples from patients with CD [13], synovial fluid and tissues from patients with inflammatory arthritis and those with RA $[67,68]$, joints of patients with spondyloarthritis [69], as well as in the peripheral blood of patients with acute-phase anti-neutrophil cytoplasmic antibody (ANCA)-associated vasculitis (AAV) [70], systemic sclerosis (SSc) [71], or systemic lupus erythematous (SLE) [72]. Individuals at risk of developing RA also

Table 1. Targeting Helper-like ILCs in Inflammatory Diseases

\begin{tabular}{|c|c|c|c|c|c|c|}
\hline Target molecule & Therapy & Drug & Target disease & Therapeutic effect & Clinical trial & Refs \\
\hline \multicolumn{7}{|l|}{ ILC1 } \\
\hline \multirow[t]{3}{*}{ IFN-Y } & \multirow[t]{3}{*}{$\mathrm{mAb}$} & \multirow[t]{2}{*}{ Fontolizumab } & $C D$ & $\begin{array}{l}\text { Decreases C-reactive protein } \\
\text { concentrations }\end{array}$ & Phase ॥ & {$[131]$} \\
\hline & & & RA & No & $\begin{array}{l}\text { Failed Phase II } \\
\text { (NCT00281294) }\end{array}$ & \\
\hline & & AMG 811 & $\begin{array}{l}\text { Discoid lupus } \\
\text { erythematosus }\end{array}$ & No & Phase I & {$[132]$} \\
\hline IL-12/23 (p40) & $\mathrm{mAb}$ & Ustekinumab & CD & Clinical benefit & Phase III & {$[133]$} \\
\hline \multicolumn{7}{|l|}{ ILC2 } \\
\hline Not defined & & $\begin{array}{l}\text { Grass pollen, } \\
\text { subcutaneous } \\
\text { immunotherapy }\end{array}$ & Seasonal allergic rhinitis & Decreases number of circulating ILC2s & & {$[85]$} \\
\hline AXL/FPR2 & $\begin{array}{l}\text { Ligand or } \\
\text { analog }\end{array}$ & Lipoxin A4 & $\begin{array}{l}\text { Asthma and allergic } \\
\text { diseases }\end{array}$ & Under evaluation $^{\mathrm{a}}$ & & {$[127]$} \\
\hline $\begin{array}{l}\text { IP (PGI2 } \\
\text { receptor) }\end{array}$ & $\begin{array}{l}\text { Ligand } \\
\text { analog }\end{array}$ & lloprost & Asthma & To be evaluated ${ }^{b}$ & & {$[128]$} \\
\hline CRTH2 & Antagonist & Fevipiprant & \multirow{6}{*}{$\begin{array}{l}\text { Asthma and allergic } \\
\text { diseases }\end{array}$} & Under evaluation & Phase III & {$[134]$} \\
\hline Leukotriene R & Antagonist & $\begin{array}{l}\text { Zafirlukast and } \\
\text { montelukast }\end{array}$ & & Under evaluation & Phase III & {$[135]$} \\
\hline TSLP & $\mathrm{mAb}$ & Tezepelumab & & Under evaluation & Phase II & {$[136]$} \\
\hline IL-13 & $\mathrm{mAb}$ & $\begin{array}{l}\text { Lebrikizumab and } \\
\text { tralokinumab }\end{array}$ & & Low effect, discontinued & Phase III & {$[136]$} \\
\hline IL-5 & $\mathrm{mAb}$ & $\begin{array}{l}\text { Mepolizumab, } \\
\text { reslizumab and } \\
\text { benralizumab }\end{array}$ & & Beneficial effects & Phase III & {$[136]$} \\
\hline $\begin{array}{l}\text { IL-4/IL-13 } \\
(\mathrm{IL}-4 \mathrm{Ra})\end{array}$ & mAb & Dupilumab & & Beneficial effects & Phase III & {$[136]$} \\
\hline \multicolumn{7}{|l|}{ ILC3 } \\
\hline IL-23R & & $\begin{array}{l}\text { Vitamin D } \\
\text { supplementation }\end{array}$ & $\mathrm{IBD}$ & Modulates IL-23R pathway & & {$[137]$} \\
\hline IL-2Ra/CD25 & $\mathrm{mAb}$ & Daclizumab & Relapsing MS & $\begin{array}{l}\text { US Food and Drug Administration } \\
\text { approved }\end{array}$ & & {$[104]$} \\
\hline IL-23 & $\mathrm{mAb}$ & Anti-IL-23 & Mouse model of colitis & Prevents and alleviates active colitis & & {$[138]$} \\
\hline IL-17A & $\mathrm{mAb}$ & Secukinumab & $\begin{array}{l}\text { Ankylosing spondylitis, } \\
\text { psoriasis, CD, and RA }\end{array}$ & Therapeutic & Phase III & [139] \\
\hline TNF- $\alpha$ & $\mathrm{mAb}$ & Anti-TNF- $a$ & Psoriasis & $\begin{array}{l}\text { Decreases inflammatory skin lesions } \\
\text { and circulating NKp } 44^{+} \text {ILC3 numbers }\end{array}$ & & {$[102]$} \\
\hline
\end{tabular}

aInhibits production of IL-13 by ILC2s and enhances ability of NK cells to induce apoptosis of eosinophils in humans.

${ }^{\mathrm{b}}$ Prevents ILC2 activation in mice. 
have high ILC1 numbers in LN biopsies relative to healthy controls [73]. An increase in ILC1 numbers at the expense of ILC2s and ILC3s might indicate the conversion of ILC2s and ILC3s into ILC1s [12].

\section{ILC2S}

ILC2s are a major source of the Th2 cytokines IL-4, IL-5, and IL-13, implicated in allergic inflammation [2]. Thus, ILC2s have a crucial role in the pathogenesis of allergen-induced asthma, by stimulating eosinophilia and the contraction of smooth muscle cells and airway epithelial cells in response to intranasal administration of papain in the mouse [74]. Both ILC2s numbers and IL-33 concentrations are high in the bronchoalveolar lavage (BAL) of patients with asthma $[75,76]$, and correlate with poor respiratory function [77]. Some ILC2s can persist long after the resolution of inflammation, and these 'allergen-experienced' cells can respond to unrelated allergens more potently compared with naïve ILC2s, displaying properties similar to those of memory $\mathrm{CD}^{+} \mathrm{T}$ cells, as observed by similarities in their gene profiling, such as vigorous proliferative and cytokine secretion responses during a secondary challenge $[78,79]$. The presence of these nonspecific antigen memory-like innate immune cells may contribute to explaining the sensitization of many patients with asthma to multiple allergens [79]. ILC2s may also have an important role in allergic asthma by promoting early-life Th2 cell skewing in the developing lungs in early childhood, at least in mice; indeed, ILC2s are recruited to developing mouse lungs from the first days of life [80-82].

Large numbers of IL-25- and IL-33-responsive ILC2s are also recovered from the nasal polyps of patients with chronic rhinosinusitis $[12,83]$ and allergic rhinitis $[84,85]$. The BAL and peripheral blood of patients with pulmonary fibrosis and interstitial lung involvement also contain significantly more ILC2s than those from controls $[86,87]$, driving the differentiation of macrophages toward a profibrotic phenotype and inducing collagen deposition by fibroblasts $[86,88,89]$. Moreover, in a mouse model of atopic dermatitis (AD) and in the skin lesions of patients with AD, large numbers of Th2 cytokine-producing ILC2s are recovered from the dermis and suction blisters $[90,91]$.

Finally, allergic sensitization to foods in the mouse is favored by ILC2s and ILC2-associated cytokines [92-94]. Indeed, oral antigen challenge of mice sensitized to OVA in tape-stripped skin resulted in IgE-dependent systemic anaphylaxis, but not in II1r1-deficient mice lacking ILC2S [92]. In another study, results showed that irradiated mice reconstituted with Rora-deficient bone marrow cells had increased resistance to experimental food allergy when sensitized with OVA (gavage fed) relative to controls [93]. Finally, mice exhibiting a gain of function mutation in IL-4R (I/4raF709 mice) were more prone to food allergy compared with WT mice, in an St2dependent manner [94]. I/4raF709 mice that lack ILC2s (II4raF709/IIr1-double deficient mice) were resistant to peanut allergy, but transfer of IL-4 producing-ILC2 was sufficient to restore anaphylaxis by reducing allergen-specific Treg numbers [94]. Mast cells, a major source of vasoactive mediators of anaphylaxis, arise at the same mucosal sites as ILC2s following exposure to food allergens in humans and mice [95]. Mast cells can promote ILC2 activation and IL-13 production by releasing the lipid mediator prostaglandin $D_{2}\left(P G D_{2}\right)$ and leukotriene $D_{4}\left(L T D_{4}\right)$, increasing the sensitivity of target tissues to mast cell mediators [96]. Collectively, these studies suggest that ILC2s regulate the severity of anaphylactic shock in response to food allergens.

ILC3S

ILC3s secrete proinflammatory or anti-inflammatory cytokines, such as IL-17 and IL-22. Whereas IL-22 promotes tissue repair [97], IL-22 may have proinflammatory effects in inflammatory diseases [98,99], as discussed herein. 
Inflammatory ILC3 numbers are high in the inflamed intestinal tissues of patients with inflammatory bowel disease (IBD) and produce more IL-22 than those from healthy controls [100]. IBD results from incompletely resolved acute colitis that develops into a chronic relapsing inflammatory disease. ILC3s also have an active role in chronic inflammatory diseases. For instance, Rora ${ }^{-/-}$ bone marrow transplant mice exhibited defective production of IL-17 and IL-22 by ILC3s and were protected from fibrosis in a model of pathogen-induced chronic gut inflammation mimicking CD [99]. Moreover, the pathogenic effects of ILC3-derived IL-22, IL-17, and IFN-Y have been described in a mouse model of ulcerative colitis that develops in anti-CD40-treated mice [98].

The skin and peripheral blood of patients with psoriasis contain larger numbers of NKp44 ${ }^{+}$ILC3s than those of patients with AD or healthy volunteers, and NKp44+ ILC3 frequency in the skin has correlated with disease severity $[101,102]$. These data suggest that NKp44 ILC3s contribute to skin pathogenesis. ILC3s were a major source of IL-17 and IL-22, inducing psoriatic plaque formation, in a mouse model of psoriasis comprising the subcutaneous injection of IL-23 [103]. In this recent report, scientists performed scRNAseq analysis of ILCs in healthy and psoriatic skin and demonstrated ILC tissue remodeling in situ, as well as conversion of tissue-resident IL-5producing ILC2s into IL-17/IL-22-producing ILC3-like cells [103].

From another angle, patients with multiple sclerosis (MS), an inflammatory disease of the central nervous system, also have large numbers of ILC3s in the blood; these cells are thought to initiate and sustain focal aggregate formation in the cerebrospinal fluid [104]. Finally, increased IL-17Aproducing lung ILC3s have been noted in a mouse model of obesity-induced asthma [105], and are more frequent in human BAL fluid samples from patients with severe asthma than in samples from healthy volunteers or patients with mild asthma [4]. Furthermore, a set of sputum ILC3-specific genes was recently identified in patients with severe adult-onset asthma [106]. Thus, ILC3s might constitute a potential therapeutic target to treat asthma.

Altogether, these reports demonstrate that helper-like ILCs are involved in several inflammatory disorders, although their exact functions remain to be fully elucidated. Nevertheless, helper-like ILCs and their related mediators have emerged as major clinical targets for putatively treating allergic inflammatory diseases (Table 1).

\section{Helper-like ILCs in Cancer}

ILCs are important mediators of several inflammatory diseases and, thus, might contribute to carcinogenesis.

ILC1S

IFN-y protects against cancer development through multiple mechanisms targeting tumor cells, promoting cancer cell growth arrest, and lymphocyte polarization and differentiation [107]. However, tumor cell-derived IFN-y has been reported to have a detrimental role by promoting the expression of inhibitory ligands, such as PD-L1, in patients with melanoma, and in the B16-F10 mouse melanoma tumor model [108]. IFN-Y is the principal cytokine produced by ILC1s. Thus, Helper-like ILC1s may help to limit tumor growth in an IFN-y-dependent manner, because IFN$Y$ is associated with antitumor mechanisms [109]. Tissue-resident ILC1s are the first cells to produce IFN- $y$ in the context of viral infection, such as MCMV, and can have a crucial role in limiting replication at the initial infection site (see earlier and [8]). However, a recent study reported that ILC1s may also facilitate tumor growth $[54,65]$. Ncr ${ }^{\mathrm{Cre}} \mathrm{TgfbR/I^{f/f }}$ mice, in which TGF- $\beta$ signaling is abolished in NKp46 cells, exhibited less intILC1s and ILC1s and were more resistant to MCA-induced fibrosarcomas relative to WT mice. intILC1s and ILC1s produced larger amounts of TNF-a compared with NK cells that might promote tumor growth and facilitate metastasis, 
although further studies are warranted to evaluate this mechanism (Figure 1) [65]. These intILC1s could result from NK cell conversion within the tumor microenvironment, as discussed earlier. Altogether, future studies are needed to infer the protective versus protumoral roles of ILC1s in different tumor contexts.

ILC2S

ILC2s are involved in tissue repair $[18,19]$ and, therefore, their functions may be triggered by epithelial damage due to tumor development. Studies of mouse transplantable tumor models have suggested a protective role for ILC2s. For instance, ILC2-derived IL-5 production was associated with eosinophil accumulation in the lungs and smaller numbers of B16 melanoma tumor nodules in tumor-bearing mice [110]. IL-33 has also been associated with delayed tumor growth and an accumulation of ILC2s and eosinophils [111]. Indeed, EL4 mouse tumor cells engineered to produce IL-33 did not form a distinguishable tumor mass upon administration in WT mice, but

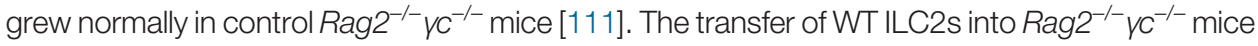
injected with EL4-producing IL-33 suppressed tumor growth and caused accumulation of eosinophils relative to saline injection [111]. Moreover, the subcutaneous growth of IL-33-expressing TC-1 tumors was slower in ILC2-deficient Rora ${ }^{-1-}$ mice than in WT mice [112]. In addition, human lung cancer tissues have abnormally low numbers of ILC2s relative to healthy control lung tissues [113]. Lastly, another study reported that ILC2s might also sense tumor cells directly, through the binding of the NCR NKp3O to its ligand, B7-H6 [114]. Specifically, both crosslinking of NKp30 via antibodies and B7H6 stimulation induced IL-13 production by human ILC2s in vitro [114].

Conversely, several studies have provided evidence against an antitumoral role of ILC2s. In mice, exogenous IL-33 promotes the primary growth and metastatic spread of 4T1 breast cancer tumors in mice, and expansion of IL-13-producing ILC2s, Treg, and myeloid-derived suppressor cell (MDSC) populations has been observed in both spleen and mammary tumors [115]. In humans, ILC2 numbers are increased in the blood of patients with gastric cancer [116] and in the urine of patients with bladder cancer following treatment with Bacillus Calmette-Guérin (BCG) [117]. Finally, IL-33 treatment can induce the accumulation of IL-13-producing ILC2s in the liver, potentially promoting tumor growth directly by favoring cholangiocyte proliferation [118]. For instance, while IL-33 treatment of WT mice induced the proliferation of cholangiocyte in the liver, this did not occur in the ILC2-deficient Rora $^{\mathbf{s g} / \mathbf{s g}}$ mouse model, where Rora is dysfunctional [118].

Thus, the role of ILC2s in promoting or preventing cancer is unclear, and different members of the ILC2 family may have different properties, some suppressing tumorigenesis, while others promote tumor growth. Therefore, future robust studies are evidently warranted.

ILC3S

ILC3s can favor the development and growth of intestinal tumors in mice, as shown by the slower tumor growth following anti-Thy1 mAb treatment that depletes ILCs in Rag1-deficient mice relative to controls [119,120]. In a mouse model of colorectal cancer (CRC), in which susceptible 129SvEv.Rag2 ${ }^{-/-}$mice were infected with Helicobacter hepaticus and treated with the carcinogen AOM, IL-22 blockade with an anti-IL-22 antibody significantly decreased tumor burden, whereas an anti-IL-17 antibody had no antitumorigenic effect, but decreased inflammation, relative to isotype control-treated mice [120]. IL-22 production can increase in human CRC tissues $[120,121]$, and this cytokine can enhance tumor development and metastasis by activating STAT3 [121]. By contrast, the presence of ILC3s in human nonsmall cell lung cancer (NSCLC) might indicate a good prognosis [113]. $\mathrm{NCR}^{+} \mathrm{ILC} 3$ s have been found in the lymphoid infiltrates 
of human NSCLC relative to healthy lung tissue from the same donor, and they produce proinflammatory, chemotactic, and angiogenic cytokines, such as TNF- $\alpha$ and IL-8 [113]. ILC3s can also interact with tumor cells and tumor-associated fibroblasts, producing TNF- $\alpha$ and IL-8 upon culture with tumor-associated fibroblasts derived from lung specimens [113]. They are more frequently detected in early-stage than in advanced-stage NSCLC, suggesting a role for $\mathrm{NCR}^{+}$ILC3S in the formation of tertiary lymphoid structures (TLSS). Indeed, the number of $\mathrm{NCR}^{+}$ILC3S in tumor infiltrates has been positively correlated with TLS density and survival [113]. In addition, the percentage of $\mathrm{NCR}^{+} \mathrm{ILC} 3 \mathrm{~s}$ in total CD45 $5^{+}$leukocytes (cells) infiltrating the tumor and their absolute number per mg of NSCLC tissue have correlated with the density of TLSs, independently of the number of leukocytes infiltrating the tumor. In addition, samples from patients with stage I and stage II NSCLC contained proportions of NCR ${ }^{+} I L C 3$ that were significantly higher than those from patients with stage III NSCLC, revealing a positive correlation between the presence of $\mathrm{NCR}^{+}$ILC3s and patient survival [113]. Collectively, these data suggest that $\mathrm{NCR}^{+}$ILC3s represent a useful prognostic factor for NSCLC, although this remains to be further assessed [113]. Altogether, these studies have suggested either pro or antitumor roles of tumor-associated ILC3s. A better understanding of the biological function of ILC3 in each particular condition is needed before new potential therapeutic approaches can be designed based on ILC3 generation and activation.

\section{Concluding Remarks}

Many studies have been performed on mammalian ILCs over the past decade since their discovery, aiming to elucidate their development and functions. Several reports have shown that ILCs might have beneficial roles in immunity and homeostasis, whereas others have highlighted their detrimental effects in various inflammatory diseases and cancers. However, patients with a selective ILC deficiency were shown to display no particular susceptibility to disease [122]. These results suggest that ILCs have redundant functions in protective immunity, provided that $\mathrm{T}$ and $\mathrm{B}$ cell functions are preserved in conditions of modern hygiene and medicine [122]. In addition, deficiencies of circulating ILCs have not been challenged in tissues, which are preferential niches for resident ILCs. Thus, the generation of specific tools, such as mouse models and antibodies, to specifically target helper-like ILCs, will contribute to the global efforts aimed at improving our understanding of their biological role (see Outstanding Questions).

Great progress has been made in the characterization of ILC development in recent years, revealing the capacities of helper-like ILCs to convert into different ILC subsets. The role and purpose of this plasticity remain unclear and the factors governing these conversions have yet to be identified (see Outstanding Questions). Still, this remarkable property of plasticity might be at the root of new putative therapeutic approaches aiming to induce a switch toward converted-ILC subsets that display non-inflammatory properties, or perhaps into those that may be favorable for tumor control.

\section{Acknowledgments}

We thank Eric Vivier for critical reading of the manuscript and mentorship. S.G. and E.N-M. are members of the laboratory of Eric Vivier, which is supported by funding from the European Research Council (ERC) under the European Union's Horizon 2020 research and innovation program (TILC, grant agreement No. 694502); the Agence Nationale de la Recherche; Equipe Labellisée 'La Ligue,' Ligue Nationale contre le Cancer, MSDAvenir, Innate Pharma and institutional grants to the CIML (INSERM, CNRS, and Aix Marseille Univ) and from Marseille Immunopole.

\section{References}

1. Spits, H. et al. (2013) Innate lymphoid cells-a proposal for uniform nomenclature. Nat. Rev. Immunol. 13, 145-149

2. Vivier, E. et al. (2018) Innate lymphoid cells: 10 years on. Cell $174,1054-1066$

\section{Outstanding Questions}

What is the biological role of helper-like ILCs? Are helper-like ILCs dispensable only in modern conditions of hygiene and medicine?

How can we specifically identify human ILCs, especially human ILC1s, in tissue sections? How can we specifically target helper-like ILC subsets in vivo to study their biological role? How can we selectively deplete helper-like ILC subsets in vivo? In particular, how can we target ILC2s and ILC2-related mediators with a major role in allergy in clinical practice?

Does helper-like ILC plasticity in situ replace ILC-poiesis? Does helper-like ILC conversion happen at steady state or is it a mechanism for adaptation to local environmental disturbances? Does helper-like ILC plasticity act in a single direction or can cells return to their initial state? In inflammatory diseases, is it possible to force helper-like ILC conversion towards a nonpathogenic state?

To which extent do mouse ILCs resemble human ILCs? Can we extrapolate ILC mouse data to humans? 
5. Gasteiger, G. et al. (2015) Tissue residency of innate lymphoid cells in lymphoid and nonlymphoid organs. Science 350, 981-985

6. Peng, H. et al. (2013) Liver-resident NK cells confer adaptive immunity in skin-contact inflammation. J. Clin. Invest. 123, 1444-1456

7. Dutton, E.E. et al. (2019) Peripheral lymph nodes contain migratory and resident innate lymphoid cell populations. Sci. Immunol. 4, eaau8082

8. Weizman, O.E. et al. (2017) ILC1 confer early host protection at initial sites of viral infection. Cell 171, 795-808

9. Mackay, L.K. et al. (2016) Hobit and Blimp1 instruct a universal transcriptional program of tissue residency in lymphocytes. Science 352, 459-463

10. Klose, C.S.N. et al. (2014) Differentiation of type 1 ILCs from a common progenitor to all helper-like innate lymphoid cell lineages. Cell 157, 340-356

11. Abt, M.C. et al. (2015) Innate immune defenses mediated by Two ILC subsets are critical for protection against acute Clostridium difficile infection. Cell Host Microbe 18, 27-37

12. Bal, S.M. et al. (2016) IL-1beta, IL-4 and IL-12 control the fate of group 2 innate lymphoid cells in human airway inflammation in the lungs. Nat. Immunol. 17, 636-645

13. Bernink, J.H. et al. (2015) Interleukin-12 and -23 control plasticity of CD127(+) Group 1 and Group 3 innate lymphoid cells in the intestinal lamina propria. Immunity 43, 146-160

14. Dahlgren, M.W. et al. (2019) Adventitial stromal cells define Group 2 innate lymphoid cell tissue niches. Immunity 50, 707-722

15. Grencis, R.K. (2015) Immunity to helminths: resistance, regulation, and susceptibility to gastrointestinal nematodes. Annu. Rev. Immunol. 33, 201-225

16. von Moltke, J. et al. (2016) Tuft-cell-derived IL-25 regulates an intestinal ILC2-epithelial response circuit. Nature 529, 221-225

17. Shimokawa, C. et al. (2017) Mast cells are crucial for induction of Group 2 innate lymphoid cells and clearance of helminth infections. Immunity 46, 863-874

18. Monticelli, L.A. et al. (2015) IL-33 promotes an innate immune pathway of intestinal tissue protection dependent on amphiregulin-EGFR interactions. Proc. Natl. Acad. Sci. U. S. A 112, 10762-10767

19. Monticelli, L.A. et al. (2011) Innate lymphoid cells promote lungtissue homeostasis after infection with influenza virus. Nat. Immunol. 12, 1045-1054

20. Krishack, P.A. et al. (2019) Protection against Staphylococcus aureus bacteremia-induced mortality depends on ILC2s and eosinophils. JCl Insight 4, e124168

21. Brestoff, J.R. et al. (2015) Group 2 innate lymphoid cells promote beiging of white adipose tissue and limit obesity. Nature 519, 242-246

22. Harms, M. and Seale, P. (2013) Brown and beige fat: development, function and therapeutic potential. Nat. Med. 19, 1252-1063

23. McKenzie, A.N.J. et al. (2014) Innate lymphoid cells in inflammation and immunity. Immunity 41, 366-374

24. Penny, H.A. et al. (2018) Orchestration of intestinal homeostasis and tolerance by group 3 innate lymphoid cells. Semin. Immunopathol. 40, 357-370

25. Ardain, A et al. (2019) Group 3 innate lymphoid cells mediate early protective immunity against tuberculosis. Nature 570 , 528-532

26. Sonnenberg, G.F. et al. (2012) Innate lymphoid cells promote anatomical containment of lymphoid-resident commensa bacteria. Science 336, 1321-1325

27. Hepworth, M.R. et al. (2013) Innate lymphoid cells regulate CD4+ T-cell responses to intestinal commensal bacteria. Nature 498, 113-117

28. Gronke, K. et al. (2019) Interleukin-22 protects intestinal stem cells against genotoxic stress. Nature 566, 249-253

29. Mortha, A. et al. (2014) Microbiota-dependent crosstalk between macrophages and ILC3 promotes intestinal homeostasis. Science 343, 1249288

30. Lim, A.I. et al. (2017) Systemic human ILC precursors provide a substrate for tissue ILC differentiation. Cell 168, 1086-1100
31. Kondo, M. et al. (1997) Identification of clonogenic common lymphoid progenitors in mouse bone marrow. Cell 91, 661-672

32. Yu, X. et al. (2014) The basic leucine zipper transcription factor NFIL3 directs the development of a common innate lymphoid cell precursor. Elife 3, e04406

33. Seillet, C. et al. (2016) Deciphering the innate lymphoid cell transcriptional program. Cell Rep. 17, 436-447

34. Harly, C. et al. (2018) Development and differentiation of early innate lymphoid progenitors. J. Exp. Med. 215, 249-262

35. Harly, C. et al. (2019) The transcription factor TCF-1 enforces commitment to the innate lymphoid cell lineage. Nat. Immunol. 20, 1150-1160

36. Yang, Q. et al. (2015) TCF-1 upregulation identifies early innate lymphoid progenitors in the bone marrow. Nat. Immunol. 16 1044-1050

37. Boos, M.D. et al. (2007) Mature natural killer cell and lymphoid tissue-inducing cell development requires Id2-mediated suppression of E protein activity. J. Exp. Med. 204, 1119-1130

38. Carotta, S. et al. (2011) Identification of the earliest NK-cell precursor in the mouse BM. Blood 117, 5449-5452

39. Renoux, V.M. et al. (2015) Identification of a human natural killer cell lineage-restricted progenitor in fetal and adult tissues. Immunity 43, 394-407

40. Constantinides, M.G. et al. (2014) A committed precursor to innate lymphoid cells. Nature 508, 397-401

41. Yu, Y. et al. (2016) Single-cell RNA-seq identifies a PD-1(hi) ILC progenitor and defines its development pathway. Nature 539 102-106

42. Walker, J.A. et al. (2019) Polychromic reporter mice reveal unappreciated innate lymphoid cell progenitor heterogeneity and elusive ILC3 progenitors in bone marrow. Immunity 51, 104-118

43. Xu, W. et al. (2019) An Id2(RFP)-reporter mouse redefines innate lymphoid cell precursor potentials. Immunity 50 1054-1068

44. Galy, A. et al. (1995) Human T, B, natural killer, and dendritic cells arise from a common bone marrow progenitor cell subset. Immunity 3, 459-473

45. Scoville, S.D. et al. (2017) Modeling human natural killer cell development in the era of innate lymphoid cells. Front. Immunol. 8,360

46. Freud, A. G. et al. (2006) Evidence for discrete stages of human natural killer cell differentiation in vivo. J. Exp. Med. 203 1033-1043

47. Scoville, S.D. et al. (2019) Cellular pathways in the development of human and murine innate lymphoid cells. Curr. Opin. Immunol. 56, 100-106

48. Scoville, S.D. et al. (2016) A progenitor cell expressing transcription factor RORgammat generates all human innate lymphoid cell subsets. Immunity 44, 1140-1150

49. Chen, L. et al. (2018) CD56 expression marks human Group 2 innate lymphoid cell divergence from a shared NK cel and Group 3 innate lymphoid cell developmental pathway. Immunity 49, 464-476

50. Vonarbourg, C. et al. (2010) Regulated expression of nuclea receptor RORgammat confers distinct functional fates to NK cell receptor-expressing RORgammat(+) innate lymphocytes. Immunity 33, 736-751

51. Cella, M et al. (2010) Expansion of human NK-22 cells with IL7, IL-2, and IL-1beta reveals intrinsic functional plasticity. Proc. Natl. Acad. Sci. U. S. A. 107, 10961-10966

52. Narni-Mancinelli, E. et al. (2011) Fate mapping analysis of lymphoid cells expressing the NKp46 cell surface receptor. Proc. Natl. Acad. Sci. U. S. A. 108, 18324-18329

53. Viant, C. et al. (2016) Transforming growth factor-beta and Notch ligands act as opposing environmental cues in regulating the plasticity of type 3 innate lymphoid cells. Sci. Signal. 9 , ra46

54. Cortez, V.S. et al. (2017) SMAD4 impedes the conversion of NK cells into ILC1-like cells by curtailing non-canonical TGF beta signaling. Nat. Immunol. 18, 995-1003

55. Cella, M. et al. (2019) Subsets of ILC3-ILC1-like cells generate a diversity spectrum of innate lymphoid cells in human mucosal tissues. Nat. Immunol. 20, 980-991 
56. Koh, J. et al. (2019) IL23-producing human lung cancer cells promote tumor growth via conversion of innate lymphoid cell 1 (ILC1) into ILC3. Clin. Cancer Res. 25, 4026-4037

57. Ohne, Y. et al. (2016) IL-1 is a critical regulator of group 2 innate lymphoid cell function and plasticity. Nat. Immunol. 17 , 646-655

58. Silver, J.S. et al. (2016) Inflammatory triggers associated with exacerbations of COPD orchestrate plasticity of group 2 innate lymphoid cells in the lungs. Nat. Immunol. 17, 626-635

59. Huang, Y. et al. (2015) IL-25-responsive, lineage-negative KLRG1(hi) cells are multipotential 'inflammatory' type 2 innate lymphoid cells. Nat. Immunol. 16, 161-169

60. Gury-BenAri, M. et al. (2016) The spectrum and regulatory landscape of intestinal innate lymphoid cells are shaped by the microbiome. Cell 166, 1231-1246

61. Seehus, C.R. et al. (2017) Alternative activation generates IL-10 producing type 2 innate lymphoid cells. Nat. Commun. 8,1900

62. Bernink, J.H. et al. (2019) c-Kit-positive ILC2s exhibit an ILC3like signature that may contribute to IL-17-mediated pathologies. Nat. Immunol. 20, 992-1003

63. Golebski, K. et al. (2019) IL-1beta, IL-23, and TGF-beta drive plasticity of human ILC2s towards IL-17-producing ILCs in nasal inflammation. Nat. Commun. 10, 2162

64. Cortez, V.S. et al. (2016) Transforming growth factor-beta signaling guides the differentiation of innate lymphoid cells in salivary glands. Immunity 44, 1127-1139

65. Gao, Y. et al. (2017) Tumor immunoevasion by the conversion of effector NK cells into type 1 innate lymphoid cells. Nat Immunol. 18, 1004-1015

66. Park, E. et al. (2019) Toxoplasma gondii infection drives conversion of NK cells into ILC1-like cells. Elife 8, e47605

67. Dalbeth, N. and Callan, M.F. (2002) A subset of natural kille cells is greatly expanded within inflamed joints. Arthritis Rheum. 46, 1763-1772

68. Dalbeth, N. et al. (2004) CD56bright NK cells are enriched at inflammatory sites and can engage with monocytes in a reciprocal program of activation. J. Immunol. 173, 6418-6426

69. Yeremenko, N. et al. (2015) AB0049 human type 1 innate lymphoid cells accumulate in the inflamed synovium in spondyloarthritis. Ann. Rheum. Dis. 74, 906

70. Braudeau, C. et al. (2016) Persistent deficiency of circulating mucosal-associated invariant T (MAIT) cells in ANCA associated vasculitis. J. Autoimmun. 70, 73-79

71. Roan, F. et al. (2016) CD4+ Group 1 innate lymphoid cells (ILC) form a functionally distinct ILC subset that is increased in systemic sclerosis. J. Immunol. 196, 2051-2062

72. Schepis, D. et al. (2009) Increased proportion of CD56bright natural killer cells in active and inactive systemic lupus erythematosus. Immunology 126, 140-146

73. Rodriguez-Carrio, J. et al. (2017) Brief Report: altered innate lymphoid cell subsets in human lymph node biopsy specimens obtained during the at-risk and earliest phases of rheumatoid arthritis. Arthritis Rheumatol. 69, 70-76

74. Halim, T.Y. et al. (2014) Group 2 innate lymphoid cells are critical for the initiation of adaptive Thelper 2 cell-mediated allergic lung inflammation. Immunity 40, 425-435

75. Bartemes, K.R. et al. (2014) Enhanced innate type 2 immune response in peripheral blood from patients with asthma. J. Allergy Clin. Immunol. 134, 671-678

76. Smith, S.G. et al. (2016) Increased numbers of activated group 2 innate lymphoid cells in the airways of patients with severe asthma and persistent airway eosinophilia. J. Allergy Clin. Immunol. 137, 75-86

77. Liu, T. et al. (2015) Type 2 innate lymphoid cells: a novel biomarker of eosinophilic airway inflammation in patients with mild to moderate asthma. Respir. Med. 109, 1391-1396

78. Martinez-Gonzalez, I. et al. (2016) Allergen-experienced Group 2 innate lymphoid cells acquire memory-like properties and enhance allergic lung inflammation. Immunity 45, 198-208

79. Martinez-Gonzalez, I. et al. (2017) Immunological memory of Group 2 innate lymphoid cells. Trends Immunol. 38, 423-431

80. de Kleer, I.M. et al. (2016) Perinatal activation of the interleukin33 pathway promotes type 2 immunity in the developing lung. Immunity 45, 1285-1298
81. Saluzzo, S. et al. (2017) First-breath-induced type 2 pathways shape the lung immune environment. Cell Rep. 18, 1893-1905

82. Steer, C.A. et al. (2017) Group 2 innate lymphoid cell activation in the neonatal lung drives type 2 immunity and allergen sensitization. J. Allergy Clin. Immunol. 140, 593-595

83. Mjosberg. J.M. et al. (2011) Human IL-25- and IL-33 responsive type 2 innate lymphoid cells are defined by expression of CRTH2 and CD161. Nat. Immunol. 12, 1055-1062

84. Fan, D.C. et al. (2016) Suppression of immunotherapy on Group 2 innate lymphoid cells in allergic rhinitis. Chin. Med. J. 129, 2824-2828

85. Lao-Araya, M. et al. (2014) Seasonal increases in peripheral innate lymphoid type 2 cells are inhibited by subcutaneous grass pollen immunotherapy. J. Allergy Clin. Immunol. 134, 1193-1195

86. Hams, E. et al. (2014) IL-25 and type 2 innate lymphoid cells induce pulmonary fibrosis. Proc. Natl. Acad. Sci. U. S. A. 111, 367-372

87. Wohlfahrt, T. et al. (2016) Type 2 innate lymphoid cell counts are increased in patients with systemic sclerosis and correlate with the extent of fibrosis. Ann. Rheum. Dis. 75, 623-626

88. Hams, E. et al. (2015) Macrophage and innate lymphoid cell interplay in the genesis of fibrosis. Front. Immunol. 6, 597

89. Li, J. et al. (2014) Biliary repair and carcinogenesis are mediated by IL-33-dependent cholangiocyte proliferation. J. Clin. Invest. 124, 3241-3251

90. Roediger, B. et al. (2013) Cutaneous immunosurveillance and regulation of inflammation by group 2 innate lymphoid cells. Nat. Immunol. 14, 564-573

91. Salimi, M. et al. (2013) A role for IL-25 and IL-33-driven type-2 innate lymphoid cells in atopic dermatitis. J. Exp. Med. 210, 2939-2950

92. Galand, C. et al. (2016) IL-33 promotes food anaphylaxis in epicutaneously sensitized mice by targeting mast cells. J. Allergy Clin. Immunol. 138, 1356-1366

93. Lee, J.B. et al. (2016) IL-25 and CD4(+) TH2 cells enhance type 2 innate lymphoid cell-derived IL-13 production, which promotes IgE-mediated experimental food allergy. J. Allergy Clin. Immunol. 137, 1216-1225

94. Noval Rivas, M. et al. (2016) IL-4 production by group 2 innate lymphoid cells promotes food allergy by blocking regulatory T-cell function. J. Allergy Clin. Immunol. 138, 801-811

95. Galli, S.J. and Tsai, M. (2012) IgE and mast cells in allergic disease. Nat. Med. 18, 693-704

96. Burton, O.T. et al. (2018) lgE promotes type 2 innate lymphoid cells in murine food allergy. Clin. Exp. Allergy 48, 288-296

97. Neurath, M.F. (2014) Cytokines in inflammatory bowel disease. Nat. Rev. Immunol. 14, 329-342

98. Eken, A. et al. (2014) IL-23R+ innate lymphoid cells induce colitis via interleukin-22-dependent mechanism. Mucosal Immunol. 7, 143-154

99. Lo, B.C. et al. (2016) The orphan nuclear receptor ROR alpha and group 3 innate lymphoid cells drive fibrosis in a mouse model of Crohn's disease. Sci. Immunol. 1, eaaf8864

100. Geremia, A. et al. (2011) IL-23-responsive innate lymphoid cells are increased in inflammatory bowel disease. J. Exp. Med. 208 1127-1133

101. Teunissen, M.B.M. et al. (2014) Composition of innate lymphoid cell subsets in the human skin: enrichment of $\mathrm{NCR}(+)$ ILC3 in lesional skin and blood of psoriasis patients. J. Invest. Dermatol. 134, 2351-2360

102. Villanova, F. et al. (2014) Characterization of innate lymphoid cells in human skin and blood demonstrates increase of NKp44+ ILC3 in psoriasis. J. Invest. Dermatol. 134, 984-991

103. Bielecki, P. et al. (2018) Skin inflammation driven by differentiation of quiescent tissue-resident ILCs into a spectrum of pathogenic effectors. bioRxiv Published online November 12 , 2018. https://doi.org/10.1101/461228

104. Perry, J.S. et al. (2012) Inhibition of LTi cell development by CD25 blockade is associated with decreased intratheca inflammation in multiple sclerosis. Sci. Transl. Med. 4, 145 ra106

105. Kim, H.Y. et al. (2014) Interleukin-17-producing innate lymphoid cells and the NLRP3 inflammasome facilitate obesityassociated airway hyperreactivity. Nat. Med. 20, 54-61 
106. Hekking, P.P. et al. (2018) Pathway discovery using transcriptomic profiles in adult-onset severe asthma. J. Allergy Clin. Immunol. 141, 1280-1290

107. Lin, C.F. et al. (2017) Escape from IFN-gamma-dependent immunosurveillance in tumorigenesis. J. Biomed. Sci. 24, 10

108. Benci, J.L. et al. (2019) Opposing functions of interferon coordinate adaptive and innate immune responses to cancer immune checkpoint blockade. Cell 178, 933-948

109. Castro, F. et al. (2018) Interferon-gamma at the crossroads of tumor immune surveillance or evasion. Front. Immunol. 9, 847

110. Ikutani, M. et al. (2012) Identification of innate IL-5-producing cells and their role in lung eosinophil regulation and antitumo immunity. J. Immunol. 188, 703-713

111. Kim, J. et al. (2016) Intratumorally establishing type 2 innate lymphoid cells blocks tumor growth. J. Immunol. 196, 2410-2423

112. Saranchova, I. et al. (2018) Type 2 innate lymphocytes actuate immunity against tumours and limit cancer metastasis. Sci. Rep. 8, 2924

113. Carrega, P. et al. (2015) NCR(+)ILC3 concentrate in human lung cancer and associate with intratumoral lymphoid structures. Nat. Commun. 6, 8280

114. Salimi, M. et al. (2016) Group 2 innate lymphoid cells express functional NKp30 receptor inducing type 2 cytokine production. J. Immunol. 196, 45-54

115. Jovanovic, I.P. et al. (2014) Interleukin-33/ST2 axis promotes breast cancer growth and metastases by facilitating intratumoral accumulation of immunosuppressive and innate lymphoid cells. Int. J. Cancer 134, 1669-1682

116. Bie, Q. et al. (2014) Polarization of ILC2s in peripheral blood might contribute to immunosuppressive microenvironment in patients with gastric cancer. J Immunol Res 2014, 923135

117. Chevalier, M.F. et al. (2017) ILC2-modulated T cell-to-MDSC balance is associated with bladder cancer recurrence. J. Clin. Invest. 127, 2916-2929

118. Li, D. et al. (2014) IL-33 promotes ST2-dependent lung fibrosis by the induction of alternatively activated macrophages and innate lymphoid cells in mice. J. Allergy Clin. Immunol. 134 1422-1432

119. Chan, I.H. et al. (2014) Interleukin-23 is sufficient to induce rapid de novo gut tumorigenesis, independent of carcinogens, through activation of innate lymphoid cells. Mucosal Immunol. 7, 842-856

120. Kirchberger, S. et al. (2013) Innate lymphoid cells sustain colon cancer through production of interleukin-22 in a mouse model. J. Exp. Med. 210, 917-931

121. Jiang, R. et al. (2013) IL-22 is related to development of human colon cancer by activation of STAT3. BMC Cancer 13, 59

122. Vely, F. et al. (2016) Evidence of innate lymphoid cell redundancy in humans. Nat. Immunol. 17, 1291-1299

123. Lunemann, S. et al. (2017) Hobit expression by a subset of human liver-resident CD56(bright) Natural Killer cells. Sci. Rep. 7, 6676
124. Freud, A.G. et al. (2016) NKp80 defines a critical step during human natural killer cell development. Cell Rep. 16, 379-391

125. Simoni, Y. et al. (2017) Human innate lymphoid cell subsets possess tissue-type based heterogeneity in phenotype and frequency. Immunity 46, 148-161

126. Li, B.W.S. et al. (2017) Group 2 innate lymphoid cells exhibit a dynamic phenotype in allergic airway inflammation. Front. Immunol. 8, 1684

127. Barnig, C. et al. (2013) Lipoxin A4 regulates natural killer cell and type 2 innate lymphoid cell activation in asthma. Sci. Transl. Med. 5, 174ra26

128. Zhou, W. et al. (2016) Prostaglandin I2 signaling and inhibition of Group 2 innate lymphoid cell responses. Am. J. Respir. Crit. Care Med. 193, 31-42

129. Yamaguchi, T. et al. (2015) Along the axis between Type 1 and Type 2 immunity; principles conserved in evolution from fish to mammals. Biology (Basel) 4, 814-859

130. Kotas, M.E. and Locksley, R.M. (2018) Why innate lymphoid cells? Immunity 48, 1081-1090

131. Reinisch, W. et al. (2010) Fontolizumab in moderate to severe Crohn's disease: a phase 2, randomized, double-blind, placebo-controlled, multiple-dose study. Inflamm. Bowel Dis. 16, 233-242

132. Werth, V.P. et al. (2017) Brief Report: Pharmacodynamics, safety, and clinical efficacy of AMG 811, a human antiinterferon-gamma antibody, in patients with discoid lupus erythematosus. Arthritis Rheumatol. 69, 1028-1034

133. Biemans, V.B.C. et al. (2019) Ustekinumab for Crohn's disease: results of the ICC Registry, a nationwide prospective observational cohort study. J. Crohns Colitis 14, 33-45

134. Issahaku, A.R. et al. (2019) Same target, different therapeutic outcome: the case of CAY10471 and fevipiprant on CRTh2 receptor in treatment of allergic rhinitis and asthma. Comb. Chem. High Throughput Screen. 22, 521-533

135. Doherty, T.A. et al. (2013) Lung type 2 innate lymphoid cells express cysteinyl leukotriene receptor 1 , which regulates TH2 cytokine production. J. Allergy Clin. Immunol. 132 205-213

136. Edris, A. et al. (2019) Monoclonal antibodies in type 2 asthma: systematic review and network meta-analysis. Respir. Res. 20 , 179

137. Konya, V. et al. (2018) Vitamin D downregulates the IL-23 receptor pathway in human mucosal group 3 innate lymphoid cells. J. Allergy Clin. Immunol. 141, 279-292

138. Elson, C.O. et al. (2007) Monoclonal anti-interleukin 23 reverses active colitis in a T cell-mediated model in mice. Gastroenterology 132, 2359-2370

139. Baeten, D. et al. (2013) Anti-interleukin-17A monoclonal antibody secukinumab in treatment of ankylosing spondylitis: a randomised, double-blind, placebo-controlled trial. Lancet 382, 1705-1713 